\title{
THE FIRST CHARACTERIZED CARBAPENEM- RESISTANT BACTEROIDES FRAGILIS STRAIN FROM CROATIA AND THE CASE STUDY FOR IT
}

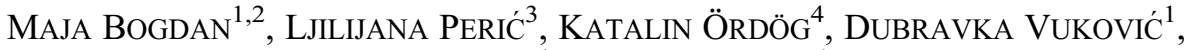 \\ EDIT URBÁN ${ }^{4}$ and JÓZSEF SÓKI ${ }^{4 *}$ \\ ${ }^{1}$ Department of Microbiology, Institute of Public Health for Osijek-Baranja County, \\ Osijek, Croatia \\ ${ }^{2}$ Faculty of Medicine, Department of Microbiology and Parasitology, University of \\ Osijek, Osijek, Croatia \\ ${ }^{3}$ Department of Infectious Diseases, Clinical Hospital Centre Osijek, Osijek, Croatia \\ ${ }^{4}$ Faculty of Medicine, Institute of Clinical Microbiology, University of Szeged, \\ Szeged, Hungary
}

(Received: 4 January 2018; accepted: 2 March 2018)

\begin{abstract}
An imipenem-resistant Bacteroides fragilis strain was isolated from the blood of a 72-year-old male patient with a urinary bladder tumor in Osijek, Croatia. This strain was also resistant to ampicillin, piperacillin/tazobactam, cefoxitin, clindamycin, tetracycline, and harbored cfiA, ermF, and tet $Q$ genes where the high-level expression of the $c f i A$ carbapenem-resistant gene was driven by an IS 1187 element. Interestingly, despite the carbapenem-resistant feature of the $B$. fragilis from blood, the patient relatively easily recovered from the bacteremia. It was the first characterized imipenem-resistant B. fragilis isolate with its case report from Croatia, which confirmed the appearance of carbapenem-resistant $B$. fragilis strains, that continues worldwide with low incidence and the molecular characteristics vary temporally and geographically.
\end{abstract}

Keywords: B. fragilis, carbapenem resistance, ertapenem, imipenem, IS 1187

\section{Introduction}

Bacteroides fragilis is the most commonly isolated opportunistic anaerobic pathogen, which is also a member of the normal microbiota. It comprizes $60 \%$ $80 \%$ of the isolated anaerobic pathogens, but gives a Bacteroides count of only $0.5 \%-1 \%$ in the gut. The infections caused vary from mild to life-threatening conditions, such as diarrhea, abdominal abscessi, other superficial soft-tissue infections, or sepsis. As a member of its parent genus, the Bacteroides, it is

*Corresponding author; E-mail: soki.jozsef@med.u-szeged.hu 
highly resistant to antibiotics regarding both the prevalence and the number of resistance mechanisms. B. fragilis is often resistant to regular $\beta$-lactams (penicillins and cephalosporins) (about 99\%) and tetracyclines (about 70\%). It may be resistant (about 10\%-30\%) to cephamycins (cefoxitin), clindamycin, or moxifloxacin. The best available antibiotics are $\beta$-lactam/ $\beta$-lactamase inhibitor combinations, carbapenems, metronidazole, or tigecycline. Its carbapenem resistance is mediated by a metallo- $\beta$-lactamase, which is coded by the cfiA gene [1]. This gene is present to a significant extent in the $B$. fragilis population, of which a distinct subgroup of the species can be identified by molecular methods. To be highly carbapenem-resistant, an insertion sequence (IS) element is necessary whose role is to provide a strong promoter for expression [2]. As we mentioned, the prevalence of true carbapenem-resistant $B$. fragilis strains is low, but such strains might still pose a serious treatment problem, if it is unrecognized [3]. The involvement of IS elements in driving the phenotypic expression of other antibiotic-resistant genes [cep $A$ - penicillin, cephalosprorin, $c f x A$ - cephamycin, erm $F$ - clindamycin, and nim - metronidazole] different from cfiA is well known for clinical Bacteroides strains [1].

In this report, we describe a case and characterization of an imipenemresistant B. fragilis strain isolated at the Institute of Public Health for Osijek-Baranja County, Osijek, Croatia. Resistance to carbapenems among Bacteroides spp. isolates was previously reported in Croatia by Novak et al. [4], but the mechanisms of resistance were not characterized or described.

\section{Materials and Methods}

The Bacteroides strains $(n=15)$ in this study were isolated from clinical specimens at the Institute of Public Health for Osijek Baranja County, Osijek, Croatia, they were identified and cultured by routine methods [5], and their diagnostic antibiotic susceptibility measurements were performed by the ATB AN $^{\circledR}$ System (bioMerieux, France). For long-term storage of the strains, their brain-heart infusion cultures containing $20 \%$ glycerol were stored at $-70{ }^{\circ} \mathrm{C}$. After sending the strains to the Institute of Clinical Microbiology, University of Szeged, Szeged, Hungary and ESCMID Study Group on Anaerobic Infections (ESGAI) cooperation, their species identities and associated genetic division of $B$. fragilis were analyzed by MALDI-TOF MS (Microflex, Bruker Daltonics, Bremen, Germany) [6]. Antibiotic susceptibility confirmation of $B$. fragilis $\mathrm{O} / 13$ (the only imipenem-resistant strain belonging to genetic Division II) was performed by the E-test method, as described by bioMeriux. Susceptibility/resistance categorization was carried out according to the EUCAST (http://www.eucast.org/clinical_breakpoints/) recommendations and 
since they are not available for moxifloxacin and tigecycline, we used the data from Eitel et al. [7].

The presence of antibiotic-resistant genes (cepA, cfxA, cfiA, nim, ermF, $\operatorname{bex} A, \operatorname{tet} Q, \operatorname{tet} X$, and $\operatorname{tet} X I)$ and their activation IS elements was detected as described earlier [8]. In brief, DNA templates were prepared by the boiling method and RT-PCR was carried out in $10 \mu \mathrm{l}$ final volumes using $5 \mu$ l of Brilliant II RTPCR master mix with SybrGREEN and ROX dyes (Agilent, Santa Clara, CA, USA), $0.7 \mu \mathrm{M}$ of primers, and $1 \mu \mathrm{l}$ of DNA templates with the primers and cycling conditions described earlier, using a StepOne RT-PCR instrument (LifeTechnologies). The harboring of the enterotoxin gene ( $b f t$ ) was detected in a similar way as the antibiotic-resistant genes as described in [9]. The plasmid content was examined using the Birnboim-Doly alkalic detergent lysis method $[9,10]$.

\section{Results}

In the period 2013-2016, antibiotic susceptibility testing using commercially available microdilution test $\mathrm{ATB} \mathrm{AN}^{\circledR}$ for 15 B. fragilis group strains was carried out at the Institute of Public Health for Osijek-Baranja County and one, namely B. fragilis $\mathrm{O} 4 / 13$, proved to be imipenem-resistant. These strains were sent to the Institute of Clinical Microbiology, University of Szeged in an ESGAI cooperation. Their species identifications were confirmed by MALDI-TOF MS and this strain also turned out to belong to Division II of $B$. fragilis by the MALDI-TOF MS project analysis (the remaining ones belonged to Division I). The case for this isolate is described as follows. The male patient (born in 1941) underwent an operation to remove a urinary bladder tumor (Dg: Adenocarcinoma vesicae urinariae) in January 2013 when a nephrostomal catheter along with permanent urinary catheter was inserted. In the following months, he regularly visited his urology specialist. During a checkup in August, he complained of a pus discharge from his nephrostomal catheter and was admitted to the infectious diseases ward at the University Hospital Osijek, Osijek, Croatia for further treatment. On admission, he was afebrile, and his blood pressure was 110/70 $\mathrm{mmHg}$, and ECG without any abnormalities (sinus rhythm, heart rate: 92 beats $/ \mathrm{min}$ ). The laboratory findings were as follows: $86.7 \mathrm{mg} / \mathrm{L}$ C-reactive protein $(\mathrm{CRP}), 10.9 \times 10^{9} / \mathrm{L}$ WBC, $2.37 \times 10^{12} / \mathrm{L} \mathrm{RBC}, 62 \mathrm{~g} / \mathrm{L}$ hemoglobin, 0.189 hematocrit, $293 \times 10^{9} / \mathrm{L}$ platelets, $25.5 \mathrm{~mol} / \mathrm{L}$ urea, $425 \mu \mathrm{mol} / \mathrm{L}$ creatinine, $136 \mathrm{mmol} / \mathrm{L} \mathrm{Na}, 5.2 \mathrm{mmol} / \mathrm{L} \mathrm{K}, 14 \mathrm{U} / \mathrm{L}$ aspartate aminotransferase (glutamate oxaloacetate transaminase), $27 \mathrm{U} / \mathrm{L}$ alanine aminotransferase (glutamate pyruvate transaminase), and $51 \mathrm{U} / \mathrm{L}$ gamma glutamyl transferase. From a urine sample collected using the left nephrostomal catheter, an extended-spectrum $\beta$-lactamase-producing Klebsiella pneumoniae was isolated. Since the patient was 
not allergic to antibiotics, along with replacement of the indwelling catheter therapy with ertapenem $1 \times 1 \mathrm{~g}$ was introduced. On the fourth day of hospitalization, the patient experienced a peak in elevated body temperature $\left(38{ }^{\circ} \mathrm{C}\right)$, so blood was collected for a blood culture set. From the inoculated anaerobic bottle, the $B$. fragilis was isolated and the aerobic bottle remained sterile. The ertapenem therapy was continued in spite of this. It is worth adding that the patient also had an anus praeter placed following radical surgery due to a malignant growth in the rectum 5 years earlier. After 7 days of ertapenem therapy and two transfusions (RBC concentrate, blood group 0, Rh-positive), the patient's health improved. At this point, his laboratory findings were as follows: $27.5 \mathrm{mg} / \mathrm{L} \mathrm{CRP}, 11.1 \times 10^{9} / \mathrm{L} \mathrm{WBC}, 3.12 / \mathrm{L}$ RBC, $92 \mathrm{~g} / \mathrm{L}$ hemoglobin, 0.277 hematocrit, $347 \times 10^{9} / \mathrm{L}$ platelets, $19 \mathrm{mmol} / \mathrm{L}$ urea, $329 \mu \mathrm{mol} / \mathrm{L}$ creatinine, $144 \mathrm{mmol} / \mathrm{L} \mathrm{Na}, 4.8 \mathrm{mmol} / \mathrm{L} \mathrm{K}$, and repeated culture of urine sample remained sterile. After resolving the acute urinary tract infection (K. pneumoniae) by ertapenem and the bacteremia probably due to the blood transfusion, he was transferred to the oncology department for continuing further treatment of developed metastatic processes. The treatment with capecitabin and irinotecan was continued and the patient was released and sent home with the recommendation of regular oncology checkups.

The $B$. fragilis $\mathrm{O} 4 / 13$ strain was further characterized by microbiological and molecular methods reported earlier. The data obtained by these methods are shown in Table I. The strain was resistant to ampicillin, piperacillin/tazobactam, cefoxitin, imipenem, clindamycin and tetracycline, intermediate-resistant to amoxicillin/ clavulanate and susceptible to metronidazole, moxifloxacin, tigecycline, and chloramphenicol. From these results, it was classed as a multidrug-resistant (MDR) isolate, since it exhibited resistance to three classes of antibiotics ( $\beta$-lactams, lincosamide, and tetracycline). However, this distinction is not so clear-cut, since the penicillin/ cephalosporin, clindamycin, and tetracycline resistances are fairly frequent among Bacteroides and Parabacteroides strains. We detected the $c f i A$ gene with an upstream, activating IS element (IS1 187) explaining the $\beta$-lactam resistances [11], an ermF gene and the common tet $Q$ gene that might have caused the tetracycline resistance. The clindamycin resistance might have been caused by the ermF gene, but we did not find the IS4351 element in its upstream region (Table I). However, such instances are known where the clindamycin resistance can be explained by the ermF gene without the well-known IS4351 [8]. No enterotoxin (fragilysin), bft, gene was detected in the strain and the only plasmid that was detected in it was a $4.1 \mathrm{~kb}$ cryptic plasmid.

\section{Discussion}

In this study, we described a carbapenem-resistant $B$. fragilis strain isolated in Osijek, Croatia, with the description of its clinical case and additionally we also 


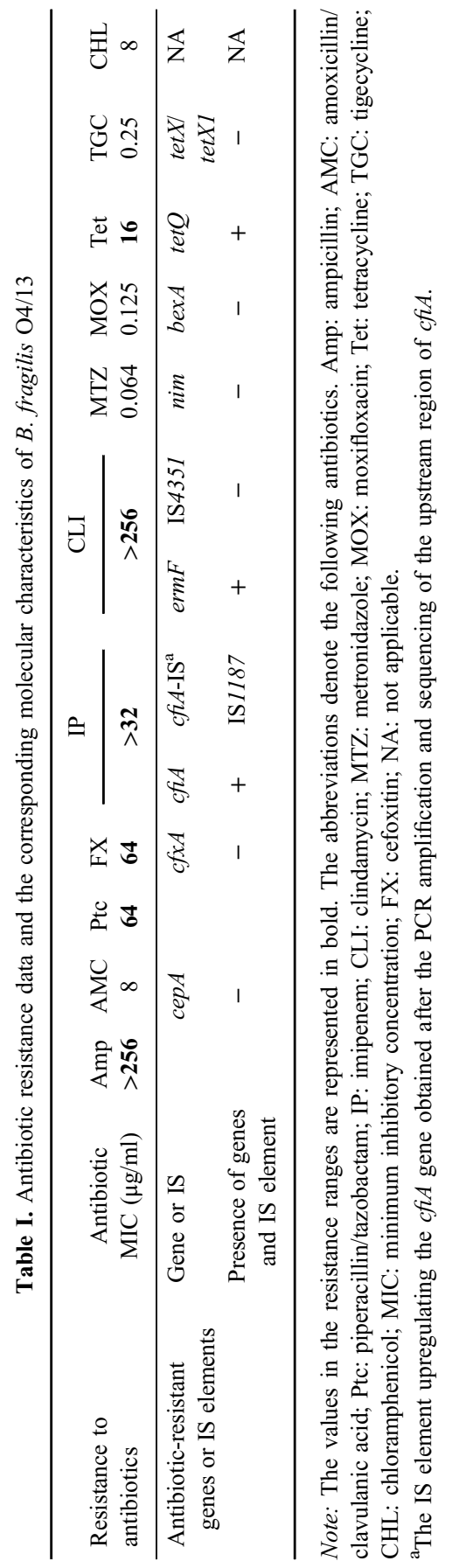


reported its microbiological and molecular biological characterizations. In this concern, we can add that true/phenotypic carbapenem resistance is still very rare among Bacteroides. However, there were numerous batch studies that reported this kind of resistance to several strains in France, Japan, USA, Hungary, Kuwait, all over Europe, Korea, Denmark, and Turkey [2, 12-17]. Several other studies reported unique strains that were isolated separately [18, 19]. For these latter strains, we also know the clinical backgrounds that were often critical to the patients harboring them. Usually, their MDR phenotypes were the major obstacles in treating the patients empirically [3]. Although the present strain isolated in Osijek, Croatia, could be regarded as MDR, the patient fortunately recovered from it with only a little help and the strain did not harbor any other significant resistance type except for some resistance to clindamycin and tetracycline. Overall, its carbapenem-resistance mechanism is consistent with our knowledge, cfiA with an IS element (IS1187) upstream, and it also indicates the spread of IS 1187, but the high clindamycin resistance (MIC $>256 \mu \mathrm{g} / \mathrm{ml}$, ermF without IS4351) requires further investigation. The characterization of this imipenemresistant $B$. fragilis strain from Osijek, Croatia, has contributed to our knowledge or at least it warns us about the occurrence, importance, and biological variability of carbapenem-resistant $B$. fragilis isolates.

\section{Acknowledgements}

This study was partly supported by the ESCMID Study Group on Anaerobic Infections (ESGAI).

\section{Conflict of Interest}

None.

\section{References}

1. Sóki, J.: Extended role for insertin sequence elements in the antibiotic resistance of Bacteroides. World J Clin Infect Dis 3, 1-12 (2013).

2. Sóki, J., Eitel, Z., Urbán, E., Nagy, E.: Molecular analysis of the carbapenem and metronidazole resistance mechanisms of Bacteroides strains reported in a Europe-wide antibiotic resistance survey. Int J Antimicrob Agents 41, 122-125 (2013).

3. Hartmeyer, G. N., Sóki, J., Nagy, E., Justesen, U. S.: Multidrug-resistant Bacteroides fragilis group on the rise in Europe? J Med Microbiol 61, 1784-1788 (2012).

4. Novak, A., Rubic, Z., Dogas, V., Goic-Barisic, I., Radic, M., Tonkic, M.: Antimicrobial susceptibility of clinically isolated anaerobic bacteria in a University Hospital Centre Split, Croatia in 2013. Anaerobe 31, 31-36 (2015). 
5. Versalovic, J., Carroll, K. C., Funke, G., Jorgensen, J. H., Landry, M. L., Warnock, D. W.: Manual of Clinical Microbiology, $10^{\text {th }}$ Edition. American Society of Microbiology, Washington, DC, 2011.

6. Fenyvesi, V. S., Urbán, E., Bartha, N., Ábrók, M., Kostrzewa, M., Nagy, E., Minarovits, J., Sóki, J.: Use of MALDI-TOF/MS for routine detection of cfiA gene-positive Bacteroides fragilis strains. Int J Antimicrob Agents 44, 474-475 (2014).

7. Eitel, Z., Sóki, J., Urbán, E., Nagy, E.: The prevalence of antibiotic resistance genes in Bacteroides fragilis group strains isolated in different European countries. Anaerobe 21, 43-49 (2013).

8. Nagy, E., Urbán, E., Nord, C. E., Bacteria on behalf of the ESCMID Study Group on Antibiotic Resistance in Anaerobic Bacteria: Antimicrobial susceptibility of Bacteroides fragilis group isolates in Europe 20 years of experience. Clin Microbiol Infect 17, 371-379 (2011).

9. Sóki, J., Eitel, Z., Terhes, G., Nagy, E., Urbán, E.: Occurrence and analysis of rare cfiA-bft doubly positive Bacteroides fragilis strains. Anaerobe 23, 70-73 (2013).

10. Sambrook, J., Fritsch, E. F., Maniatis, T.: Molecular Cloning: A Laboratory Manual. Cold Spring Harbor Laboratory Press, Cold Spring Harbor, NY, 1989, pp. 1.25-1.28.

11. Podglajen, I., Breuil, J., Rohaut, A., Monsempes, C., Collatz, E.: Multiple mobile promoter regions for the rare carbapenem resistance gene of Bacteroides fragilis. J Bacteriol 183, 3531-3535 (2001).

12. Podglajen, I., Breuil, J., Casin, I., Collatz, E.: Genotypic identification of two groups within the species Bacteroides fragilis by ribotyping and by analysis of PCR-generated fragment patterns and insertion sequence content. J Bacteriol 177, 5270-5275 (1995).

13. Kato, N., Yamazoe, K., Han, C. G., Ohtsubo, E.: New insertion sequence elements in the upstream region of cfiA in imipenem-resistant Bacteroides fragilis strains. Antimicrob Agents Chemother 47, 979-985 (2003).

14. Sydenham, T. V., Sóki, J., Hasman, H., Wang, M., Justesen, U. S.: Identification of antimicrobial resistance genes in multidrug-resistant clinical Bacteroides fragilis isolates by whole genome shotgun sequencing. Anaerobe 31, 59-64 (2015).

15. Roh, K. H., Kim, S., Kim, C.-K., Yum, J. H., Kim, M. S., Yong, D., Jeong, S. H., Lee, K., Kim, J. M., Chong, Y.: New cfiA variant and novel insertion sequence elements in carbapenem-resistant Bacteroides fragilis isolates from Korea. Diagn Microbiol Infect Dis 66, 343-348 (2010).

16. Toprak, N. U., Uzunkaya, O. D., Sóki, J., Soyletir, G.: Susceptibility profiles and resistance genes for carbapenems (cfiA) and metronidazole (nim) among Bacteroides species in a Turkish University Hospital. Anaerobe 18, 169-171 (2012).

17. Ferløv-Schwensen, S. A., Sydenham, T. V., Hansen, K. C. M., Hoegh, S. V., Justesen, U. S.: Prevalence of antimicrobial resistance and the cfiA resistance gene in Danish Bacteroides fragilis group isolates since 1973. Int J Antimicrob Agents 50, 552-556 (2017).

18. Cascio, G. L., Macaccaro, L., Centonze, A. R., Sóki, J., Fontana, R., Mazzariol, A.: A new insertion sequence element containing a cfiA gene in the first imipenem-resistant Bacteroides fragilis strain isolated in Italy. Int J Antimicrob Agents 34, 608-609 (2009).

19. Walsh, T. R., Onken, A., Haldorsen, B., Toleman, M. A., Sundsfjord, A.: Characterization of a carbapenemase-producing clinical isolate of Bacteroides fragilis in Scandinavia: Genetic analysis of a unique insertion sequence. Scand J Infect Dis 37, 676-679 (2005). 